Archive for

Organic Chemistry

Arkivoc 2020, part vi, 11-20

\title{
Desymmetrizing Hantzsch pyridines via promoter-free benzylic C-H functionalization
}

\author{
Yong-Qing Yang, Zheng Lu, ${ }^{*}$ Jinming Chen, and Ximing Xu \\ School of Pharmacy, Jiangsu University, Zhenjiang, Jiangsu, 212 013, China \\ Email: $\underline{\text { z@ujs.edu.cn }}$
}

\section{Dedicated to Xiyan Lu on the occasion of his $91^{\text {st }}$ birthday}

Received 11-21-2019

Accepted 02-12-2020

Published on line $02-28-2020$

\section{Abstract}

1,4-Dihydropyridines are an important class of calcium channel blockers. However, difficulties in the preparation of nonsymmetric Hantzsch esters impede further development in this area. Benzylic $\mathrm{C}-\mathrm{H}$ functionalization of Hantzsch pyridines is an efficient approach to nonsymmetric Hantzsch pyridines. We herein report a promoter-free benzylic $\mathrm{C}-\mathrm{H}$ functionalization of pyridines, which allows preparation of a variety of pyridines unsymmetrically substituted in the 2/6-positions. A plausible mechanism is proposed for this reaction. This work provides a new way to prepare unsymmetrical pyridines and enriches the scope of $\mathrm{C}-\mathrm{H}$ functionalization of aza-arenes.<smiles>[R]C=C(C#N)[NH2+][CH]C(=O)c1c(C)nc(C)c(C(=O)OCC)c1[R]</smiles>

- No additive required

- 12 Examples

- Yield up to $97 \%$

Keywords: Hantzsch pyridines, benzylic C-H functionalization, azaarenes, malononitriles 


\section{Introduction}

Hantzsch 1,4-dihydropyridines (1,4-DHPs) are important pharmaceuticals, which are well-known as dipines (calcium channel blockers) (Figure 1). ${ }^{1-3}$ Interestingly, most are nonsymmetric 1,4-DHPs except for a few (e.g., Nifedipine, which is symmetrical). The Hantzsch reaction ${ }^{4-7}$ is an efficient way to produce symmetric 1,4-DHPs. However, nonsymmetric 1,4-DHPs are not easy to be obtained via Hantszch reaction directly, as an enamine has to be prepared beforehand. ${ }^{8,9}$ As it has been reported that polysubstituted pyridines can be reduced directly to the corresponding 1,4 -dihydropyridines, ${ }^{10-12}$ this gives rise to new approaches to nonsymmetric $1,4-$ dihydropyridines.

Recently, two research groups ${ }^{13,14}$ have reported that the direct benzylic C-H bond functionalization of azaarenes leads to nonsymmetric pyridines bearing cyano groups (CN), which have the potential to act as a chemical handle. We are very interested in applying these methods by direct benzylic $\mathrm{C}-\mathrm{H}$ bond functionalization of Hantzsch pyridines to construct a variety of asymmetric Hantszch pyridine derivatives, which, by themselves or after reduction to asymmetric 1,4-DHPs, might show antianginal, antiarrhythmic, or antihypertensive bioactivity.

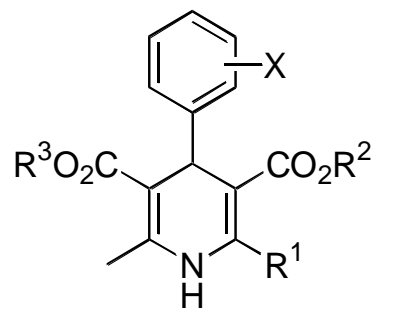

Nifedipine: $\mathrm{R}^{1}=\mathrm{CH}_{3}, \mathrm{R}^{2}=\mathrm{R}^{3}=\mathrm{CH}_{3}, \mathrm{X}=2-\mathrm{NO}_{2}$ Amlodipine: $\mathrm{R}^{1}=\mathrm{CH}_{2} \mathrm{OCH}_{2} \mathrm{CH}_{2} \mathrm{NH}_{2}, \mathrm{R}^{2}=\mathrm{CH}_{2} \mathrm{CH}_{3}, \mathrm{R}^{3}=\mathrm{CH}_{3}, \mathrm{X}=2-\mathrm{Cl}$

Nilvadipine: $\mathrm{R}^{1}=\mathrm{CN}, \mathrm{R}^{2}=\mathrm{CH}_{3}, \mathrm{R}^{3}=\mathrm{CH}\left(\mathrm{CH}_{3}\right)_{2}, \mathrm{X}=3-\mathrm{NO}_{2}$

Dihydropyridine calcium channel blockers

Figure 1. Representative 1,4-dihydropyridines as calcium channel blockers.

\section{Results and Discussion}

Initially, 1a (2-benzylidenemalononitrile) and 2a (Hantzsch pyridine) were both dissolved in PEG-400 and heated to $100{ }^{\circ} \mathrm{C}$ for $24 \mathrm{hr} .{ }^{14}$ However, no product was detected (Table 1, entry 1 ). Then if the mixture was suspended in $\mathrm{H}_{2} \mathrm{O}$ and heated at $100{ }^{\circ} \mathrm{C}$ for $10 \mathrm{hr},{ }^{13}$ the desired product $3 a$ could be obtained in $50 \%$ yield (entry 2). To further optimize the reaction conditions, we carried out further trials. Under the condition of $\mathrm{Pd} / \mathrm{C}(5 \mathrm{~mol} \%)+\mathrm{TsOH}(10 \%)$ in $\mathrm{MeOH}$ under reflux for $20 \mathrm{hr}, 59 \%$ of the desired product was achieved (entry 3). When $\mathrm{TsOH}(10 \%)$ was employed as acidic catalyst and toluene was used as solvent, $3 a$ was obtained in 60 $\%$ yield (entry 4). Interestingly, promoter-free conditions gave a slightly higher yield (entry 5). Therefore it seems that the promoter does not contribute much to the reaction; the reaction temperature is the important factor. Under promoter-free conditions we tried three different solvents at reflux. It turned out that refluxing in xylene for $10 \mathrm{hr}$ gave the best results ( $88 \%$ in $10 \mathrm{hr}$ ). See Table 1.

With the optimal conditions in hand, we were eager to know whether this reaction could tolerate different functional groups. Reactions of alkenes with different functional groups and pyridines bearing electron-withdrawing groups were carried out, and the results are listed in Table 2. 
Table 1. Screening of benzylic $\mathrm{C}-\mathrm{H}$ functionalization of Hantzsch pyridine $\mathbf{2 a}$ with electron deficient alkene $1 \mathrm{a}^{\mathrm{a}}$

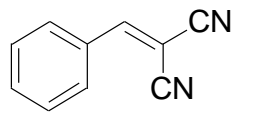

$1 \mathbf{a}$

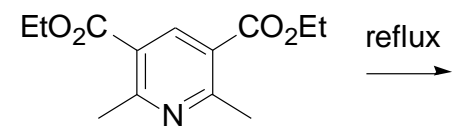

$2 a$

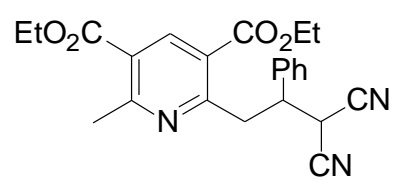

$3 a$

\begin{tabular}{ccccc}
\hline Entry & Additive & Solvent & Time/h & Yield $/ \%^{b}$ \\
\hline 1 & None & PEG-400 & 24 & - \\
2 & None & $\mathrm{H}_{2} \mathrm{O}$ & 10 & 50 \\
3 & $\mathrm{Pd} / \mathrm{C}(5 \mathrm{~mol} \%)+\mathrm{TsOH}(10 \%)$ & $\mathrm{MeOH}$ & 20 & 59 \\
4 & $\mathrm{TsOH}(10 \%)$ & Toluene & 20 & 60 \\
5 & None & Toluene & 20 & 63 \\
6 & None & MeOH & 20 & 55 \\
7 & None & xylene & 10 & 88 \\
8 & None & DCM & 24 & - \\
\hline
\end{tabular}

${ }^{\text {a }}$ Solvent $(2 \mathrm{~mL})$ was added into a reaction vessel charged with compound 1a (1.0 mmol), $2 \mathrm{a}(1.2 \mathrm{mmol})$ and additives, the vessel was purged with nitrogen and then heated under reflux over the indicated period. ${ }^{b}$ Isolated yield.

Refluxing in xylene, all of the alkenes reacted smoothly with Hantzsch pyridines 2, although the yields of the products were variable. For alkenes with a 3-aryl group, the electronic nature of the functional group at the para position affects the reaction yield. When it is an electron-donating group, the yield decreases markedly (see 3b). However, an electron withdrawing group does not affect the yield much (see $\mathbf{3 c}$ and $\mathbf{3 d}$ ). Interestingly, when alkenes with nitro group on different position of the aryl group are tested, it is found that meta-nitro substitution gives higher yield than para-nitro substitution, while a nitro group in the ortho postion makes the yield drop (see $\mathbf{3 d} \mathbf{d} \mathbf{3}$ e and $\mathbf{3 f}$ ). In terms of electron-deficient alkenes with heteroaryl group, electron donating furyl and thienyl both gave poor yields (see $\mathbf{3 h}$ and $\mathbf{3 i}$ ). We were curious to know whether an alkyl group on the alkene would retard this reaction because of its electron donating capability. $t$-Butyl was chosen as an example of alkyl group to avert any $\mathrm{C}-\mathrm{H}$ bond on the $\alpha$-position of the alkene being cleaved and generating undesired side producs. ${ }^{15-18}$ The reaction did occur although the yield is, not surprisingly, unsatisfactory. (see $\mathbf{3 j}$ ) When a Hantszch pyridine bearing a 4-phenyl group was used as the nucleophile, the addition product was isolated in reasonable yield (see 31). Furthermore, microwave irradiation was tried on reactions with poor yields (see $\mathbf{3 b}, \mathbf{3} \mathbf{g}-\mathbf{j}$ ), and yields were improved somewhat except for that of $\mathbf{3} \mathbf{j}$. It is also interesting to find that a 2-methyl is preferred to a 4-methyl for the $\mathrm{C}-\mathrm{H}$ functionalization of a Hantzsch pyridine (see 3k). With the weak electron-donating group, phenyl, on the 4-position of the Hantzsch pyridine, the outcome of the reaction is much better than that of the 4-methyl Hantzsch pyridine (see $3 \mathbf{k}$ and $3 \mathbf{3}$ ). 
Table 2. Benzylic $\mathrm{C}-\mathrm{H}$ functionalization of Hantzsch pyridines 2 with methylenemalononitriles $1^{\text {a }}$

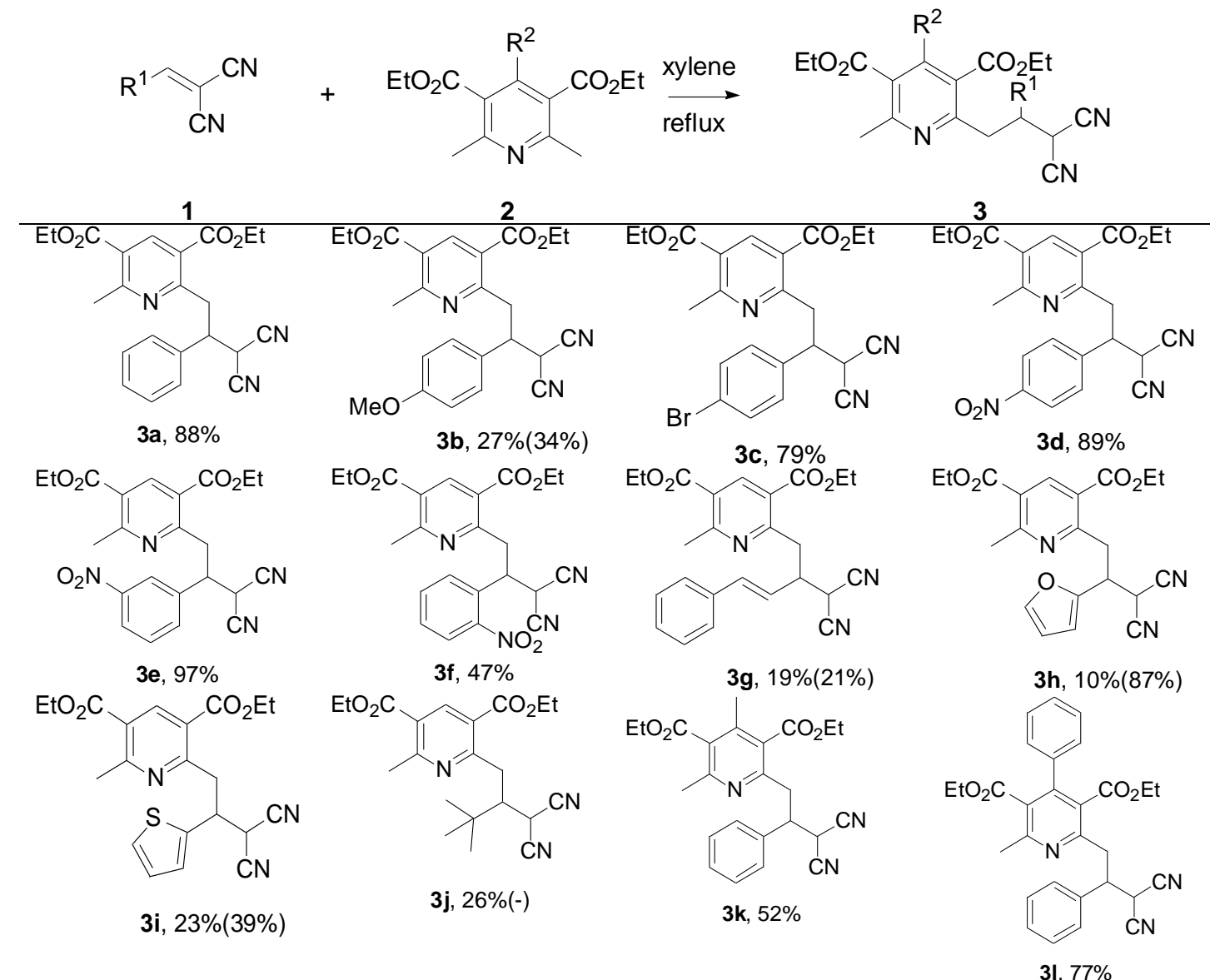

${ }^{a}$ Solvent $(2 \mathrm{~mL})$ was added into reaction vessel charged with compound 1 (1.0 $\mathrm{mmol})$ and $2(1.2 \mathrm{mmol})$, the vessel was purged with nitrogen and them heated under reflux for 10 hours. ${ }^{b}$ Isolated yield, the yield in parenthesis is that obtained under microwave irradiation for 10 hours.

To understand the mechanism of this promoter-free $\mathrm{C}-\mathrm{H}$ functionalization reaction, several easily accessible methylpyridines were subjected to these reaction conditions and the results are listed in Table 3. As observed above, a methyl in the 2-position reacts more readily than one at the 4-position, while a methyl in a 3-position does not participate in the reaction at all. 
Table 3. Benzylic C-H functionalization of methyl pyridines 4 with 2-benzylidenemalononitrile $1 a^{a}$<smiles>[R]c1ccc[R]([AlH2])n1</smiles><smiles>[R]c1cccc(C(c2ccccc2)C(C#N)C#N)n1</smiles>

1a $4 \quad 5$

\begin{tabular}{cccccc} 
& $\mathbf{1 a}$ & \multicolumn{4}{c}{$\mathbf{5}$} \\
\hline 2 & $\mathrm{R}^{1}$ & $\mathrm{R}^{2}$ & Product & Time/h & Yield/\% ${ }^{\mathrm{b}}$ \\
\hline 1 & $2-\mathrm{CH}_{3}$ & $\mathrm{CH}_{3}$ & $\mathbf{5 a}$ & 4.5 & 62 \\
2 & $4-\mathrm{CH}_{3}$ & $\mathrm{H}$ & $\mathbf{5 b}$ & 29 & 12 \\
3 & $3-\mathrm{CH}_{3}$ & $\mathrm{H}$ & & 40 & $-{ }^{\mathrm{b}}$
\end{tabular}

a Solvent $(2 \mathrm{~mL})$ was added into reaction vessel charged with compounds 1 a (1.0 $\mathrm{mmol}$ ) and 4 (1.2 mmol), the vessel was purged with nitrogen and then heated under reflux over the indicated period. ${ }^{b}$ Isolated yield. ${ }^{\mathrm{c}}$ No desired product isolated.

From the above findings, the reaction mechanism could be postulated as shown in Scheme 1 . There is an equilibrium between compound 2 and its enamine tautomer $2^{*}$, which is the Michael donor in the reaction but much less stable than ground state compound 2 . Intermediate $\mathbf{2}^{*}$ adds to electron-deficient compound $\mathbf{1}$ to give product 3 via nucleophilic conjugate addition.

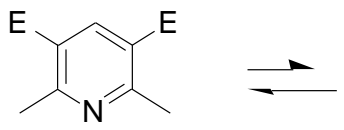

2

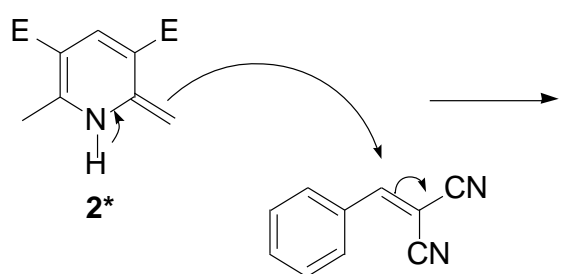

1<smiles>Cc1nc(CC(c2ccccc2)C(C#N)C#N)c(F)cc1F</smiles>

3

Scheme 1. A plausible mechanism of benzylic C-H functionalization on Hantzsch pyridine with electrondeficient alkenes.

\section{Conclusions}

A promoter-free benzylic $\mathrm{C}-\mathrm{H}$ functionalization on substituted pyridine with electron deficient alkene is found, which can tolerate a variety of pyridine substrates. Fourteen compounds have been prepared by this method, might be applied to a series of bioassay to find out interesting pharmaceutical activities. This promoter-free benzylic $\mathrm{C}-\mathrm{H}$ functionalization could be an effective tool to prepare nonsymmetric pyridine derivatives.

\section{Experimental Section}

General. The solvents used were analytical reagent purchased from Sinopharm Chemical Reagent Co, Ltd. The starting materials used were prepared in-house from chemicals purchased from Sinopharm. The reactions described in this manuscript were performed in standard laboratory glassware. Melting points were recorded on a WRS-1B digital melting point recorder from Shanghai Precision Scientific Instrument Corporation. Infrared 
spectra were recorded on a Nicolet Avatar 370 DTGS machine. ${ }^{1} \mathrm{H}$ NMR and ${ }^{13} \mathrm{C}$ NMR spectra were obtained on a Bruker AV400 equipment. MS data were recorded on a Shimadzu LCMS-2010EV mass spectrometer or an Agilent 5973N GC/MS. The HRMS were run using an Agilent 6224 TOF LC/MS or a Thermo LXQ Linear lon Trap Mass Spectrometer.

\section{General procedure (Method A) for preparation of unsymmetrical Hantzsch pyridines}

Xylene $(2 \mathrm{~mL}$ ) was added into reaction vessel charged with compound 1 (1.0 mmol) and 2 (1.2 mmol), the vessel was purged with nitrogen and then heated under reflux for $10 \mathrm{hr}$. The reaction mixture was then subjected to flash chromatography to give the required product.

\section{Alternative procedure (Method B)}

Xylene $(2 \mathrm{~mL})$ was added into reaction vessel charged with compound 1 (1.0 mmol) and 2 (1.2 mmol), the vessel was irradiated with microwave at $250 \mathrm{~W}$ for $10 \mathrm{hr}$. The reaction mixture was then subjected to flash chromatography to give the product.

2-Cyano-4-[2-(3,5-diethoxycarbonyl-6-methyl)pyridyl]-3-phenylbutyronitrile (3a). Light yellow oil (361 mg, $0.89 \mathrm{mmol}$ ), yield $88 \% . \mathrm{R}_{f}=0.36$ (PE-EtOAc, 4:1). ${ }^{1} \mathrm{H} \mathrm{NMR}\left(400 \mathrm{MHz}, \mathrm{CDCl}_{3}\right) \delta 8.75\left(\mathrm{~s}, 1 \mathrm{H}, \mathrm{Py}-\mathrm{H}-4^{\prime}\right), 7.46-7.32$ (m, 5H, Ph-H), 4.81 (d, J $5.2 \mathrm{~Hz}, 1 \mathrm{H}, \mathrm{H}-2), 4.42$ (q, J $7.2 \mathrm{~Hz}, 2 \mathrm{H}, \mathrm{OCH}_{2} \mathrm{CH}_{3}$ ), 4.39 (q, J 7.2 Hz, 2H, OCH $\left.\mathrm{CH}_{3}\right), 4.07$ (dt, J 8.8, 5.6 Hz, 1H, H-3), 3.99-3.88 (m, 2H, H-4), 2.92 (s, 3H, Py- $\mathrm{CH}_{3}-2^{\prime}$ ), 1.42 (t, J 7.2 Hz, 3H, $\left.\mathrm{CH}_{3} \mathrm{CH}_{2} \mathrm{O}\right), 1.39$ (t, J 7.2 Hz, 3H, $\left.\mathrm{CH}_{3} \mathrm{CH}_{2} \mathrm{O}\right) .{ }^{13} \mathrm{C} N M R\left(100 \mathrm{MHz}, \mathrm{CDCl}_{3}\right) \delta 165.5,165.3,162.4,160.2,141.4,137.2,129.0,128.8$, 128.2, 124.0, 123.5, 112.5, 112.0, 61.9, 61.7, 44.5, 37.8, 28.9, 25.1, 14.3, 14.2. IR (KBr) $v_{\max } 2983,2254,1720$, $1595 \mathrm{~cm}^{-1}$. MS (El, 70eV) m/z (\%) = $405\left(\mathrm{M}^{+}, 31\right), 251(100)$. HRMS-TOF: calcd for $\mathrm{C}_{23} \mathrm{H}_{24} \mathrm{~N}_{3} \mathrm{O}_{4}[\mathrm{M}+\mathrm{H}]^{+}$ 406.1761, found: 406.1759 .

2-Cyano-4-[2-(3,5-diethoxycarbonyl-6-methyl)pyridyl]-3-(4-methoxyphenyl)butyronitrile (3b). Light yellow oil, $27 \%$ (120 mg, $0.28 \mathrm{mmol}$ ); $34 \%$ (157 mg, $0.36 \mathrm{mmol}$ ) by Method B. R $\mathrm{R}_{f}=0.30$ (PE-EtOAc, 4:1). ${ }^{1} \mathrm{H}$ NMR (400 $\left.\mathrm{MHz}, \mathrm{CDCl}_{3}\right) \delta 8.72\left(\mathrm{~s}, 1 \mathrm{H}, \mathrm{Py}-\mathrm{H}-4^{\prime}\right), 7.37\left(\mathrm{~d}, J 8.8 \mathrm{~Hz}, 2 \mathrm{H}, \mathrm{Ph}-\mathrm{H}-3^{\prime} \& \mathrm{Ph}-\mathrm{H}-5^{\prime}\right), 6.92\left(\mathrm{~d}, J 8.8 \mathrm{~Hz}, 2 \mathrm{H}, \mathrm{Ph}-\mathrm{H}-2^{\prime} \& \mathrm{Ph}-\mathrm{H}-\right.$ $\left.6^{\prime}\right), 4.71(\mathrm{~d}, J 5.2 \mathrm{~Hz}, 1 \mathrm{H}, \mathrm{H}-2), 4.41\left(\mathrm{q}, J 7.2 \mathrm{~Hz}, 2 \mathrm{H}, \mathrm{OC}_{2} \mathrm{CH}_{3}\right), 4.39\left(\mathrm{q}, J 7.2 \mathrm{~Hz}, 2 \mathrm{H}, \mathrm{OCH}_{2} \mathrm{CH}_{3}\right), 4.03$ (dt, J 9.2, $5.2 \mathrm{~Hz}, 1 \mathrm{H}, \mathrm{H}-3), 3.91$ (dd, J 16.0, $5.2 \mathrm{~Hz}, 1 \mathrm{H}, \mathrm{H}-4), 3.83(\mathrm{dd}, J 15.6,9.6 \mathrm{~Hz}, 1 \mathrm{H}, \mathrm{H}-4), 3.81\left(\mathrm{~s}, 3 \mathrm{H}, \mathrm{OCH}_{3}\right), 2.89(\mathrm{~s}$, $\left.3 \mathrm{H}, \mathrm{Py}-\mathrm{CH}_{3}-6^{\prime}\right), 1.42\left(\mathrm{t}, J 7.2 \mathrm{~Hz}, 3 \mathrm{H}, \mathrm{OCH}_{2} \mathrm{CH}_{3}\right), 1.39\left(\mathrm{t}, J 7.2 \mathrm{~Hz}, 3 \mathrm{H}, \mathrm{OCH}_{2} \mathrm{CH}_{3}\right) .{ }^{13} \mathrm{C} \mathrm{NMR}(100 \mathrm{MHz}$, $\left.\mathrm{CDCl}_{3}\right) \delta 165.5,165.3,162.4,160.3,159.8,141.4,129.3,129.2,124.0,123.6,114.4,112.6,112.1,61.9,61.7$, $55.3,43.9,37.9,29.2,25.1,14.3,14.2$. IR (KBr) $v_{\max } 2983,2254,1717,1612,1595,1552 \mathrm{~cm}^{-1}$. HRMS-TOF: calcd for $\mathrm{C}_{24} \mathrm{H}_{26} \mathrm{~N}_{3} \mathrm{O}_{5}[\mathrm{M}+\mathrm{H}]^{+} 436.1867$, found: 436.1868 .

3-(4-Bromophenyl)-2-cyano-4-[2-(3,5-diethoxycarbonyl-6-methyl)pyridyl]butyronitrile (3c). Yellow oil, 79 \% (328 mg, $0.68 \mathrm{mmol}$ ). $\mathrm{R}_{f}=0.35$ (PE-EtOAc, 4:1). ${ }^{1} \mathrm{H}$ NMR (400 MHz, CDCl $) \delta 8.72\left(\mathrm{~s}, 1 \mathrm{H}, \mathrm{Py}-\mathrm{H}-4^{\prime}\right), 7.54$ (d, J 8.4 $\left.\mathrm{Hz}, 2 \mathrm{H}, \mathrm{Ph}-\mathrm{H}-3^{\prime} \& \mathrm{Ph}-\mathrm{H}-5^{\prime}\right), 7.34$ (d, J $\left.8.4 \mathrm{~Hz}, 2 \mathrm{H}, \mathrm{Ph}-\mathrm{H}-2^{\prime} \& \mathrm{Ph}-\mathrm{H}-6^{\prime}\right), 4.73(\mathrm{~d}, J 5.2 \mathrm{~Hz}, 1 \mathrm{H}, \mathrm{H}-2), 4.42(\mathrm{q}, J 7.2 \mathrm{~Hz}$, $\left.2 \mathrm{H}, \mathrm{OC}_{2} \mathrm{CH}_{3}\right), 4.39$ (q, J 7.2 Hz, 2H, OC$\left.{ }_{2} \mathrm{CH}_{3}\right), 4.06$ (dt, J 9.2, 5.6 Hz, 1H, H-3), 3.91 (dd, J 16.0, 5.6 Hz, 1H, H-4), 3.82 (dd, J 16.0, 9.2 Hz, 1H, H-4), 2.88 (s, 3H, Py- $\left.\mathrm{CH}_{3}-6^{\prime}\right), 1.43\left(\mathrm{t}, J 7.2 \mathrm{~Hz}, 3 \mathrm{H}, \mathrm{OCH}_{2} \mathrm{CH}_{3}\right), 1.40(\mathrm{t}, J 7.2 \mathrm{~Hz}, 3 \mathrm{H}$, $\left.\mathrm{OCH}_{2} \mathrm{CH}_{3}\right) .{ }^{13} \mathrm{C}$ NMR $\left(100 \mathrm{MHz}, \mathrm{CDCl}_{3}\right) \delta 165.3,165.0,162.3,159.7,141.3,136.1,132.0,129.8,123.9,123.3$, 122.7, 112.2, 111.7, 61.8, 61.6, 43.8, 37.4, 28.6, 24.9, 14.1, 14.1. IR (KBr) $v_{\max } 2983,2905,2255,1719,1594$, $1552 \mathrm{~cm}^{-1}$. HRMS-TOF: calcd for $\mathrm{C}_{23} \mathrm{H}_{23} \mathrm{BrN}_{3} \mathrm{O}_{4}[\mathrm{M}+\mathrm{H}]^{+} 484.0866$, found: 484.0864 .

2-Cyano-4-[2-(3,5-diethoxycarbonyl-6-methyl)pyridyl]-3-(4-nitrophenyl)butyronitrile (3d). Light brown oil, 89 \% (448 mg, $0.99 \mathrm{mmol}$ ). $\mathrm{R}_{f}=0.35$ (PE-EtOAc, 3:1). ${ }^{1} \mathrm{H}$ NMR $\left(400 \mathrm{MHz}, \mathrm{CDCl}_{3}\right) \delta 8.78\left(\mathrm{~s}, 1 \mathrm{H}, \mathrm{Py}-\mathrm{H}-4^{\prime}\right), 8.29(\mathrm{~d}, J$ $\left.8.8 \mathrm{~Hz}, 2 \mathrm{H}, \mathrm{Ph}-\mathrm{H}-3^{\prime} \& \mathrm{Ph}-\mathrm{H}-5^{\prime}\right), 7.69$ (d, J $\left.8.8 \mathrm{~Hz}, 2 \mathrm{H}, \mathrm{Ph}-\mathrm{H}-2^{\prime} \& \mathrm{Ph}-\mathrm{H}-6^{\prime}\right), 4.88$ (d, J $\left.5.2 \mathrm{~Hz}, 1 \mathrm{H}, \mathrm{H}-2\right), 4.43$ (q, J 7.2 $\left.\mathrm{Hz}, 2 \mathrm{H}, \mathrm{OCH}_{2} \mathrm{CH}_{3}\right), 4.41\left(\mathrm{q}, J 7.2 \mathrm{~Hz}, 2 \mathrm{H}, \mathrm{OCH}_{2} \mathrm{CH}_{3}\right), 4.25(\mathrm{dt}, J$ 8.8, $5.6 \mathrm{~Hz}, 1 \mathrm{H}, \mathrm{H}-3), 3.99(\mathrm{dd}, J 16.0,5.6 \mathrm{~Hz}, 1 \mathrm{H}$, 
$\mathrm{H}-4$ ), 3.93 (dd, J 16.4, 9.2 Hz, 1H, H-4), 2.91 (s, 3H, Py- $\mathrm{CH}_{3}-6^{\prime}$ ), 1.43 (t, J 7.2 Hz, 3H, OCH $\mathrm{CH}_{3}$ ), 1.42 (t, J 7.2 Hz, $\left.3 \mathrm{H}, \mathrm{OCH}_{2} \mathrm{C}_{3}\right) .{ }^{13} \mathrm{C} \mathrm{NMR}\left(100 \mathrm{MHz}, \mathrm{CDCl}_{3}\right) \delta 165.3,165.0,162.4,159.2,147.9,144.4,141.4,129.4,124.2,124.0$, $123.3,112.0,111.5,61.8,61.7,43.9,37.3,28.4,24.9,14.11,14.10 . \mathrm{IR}(\mathrm{KBr}) v_{\max } 3082,2984,2907,2256,1721$, 1596, $1525 \mathrm{~cm}^{-1}$. HRMS-TOF: calcd for $\mathrm{C}_{23} \mathrm{H}_{23} \mathrm{~N}_{4} \mathrm{O}_{6}[\mathrm{M}+\mathrm{H}]^{+} 451.1612$, found: 451.1611 .

2-Cyano-4-[2-(3,5-diethoxycarbonyl-6-methyl)pyridyl]-3-(3-nitrophenyl)butyronitrile (3e). Off-white solid, 97 $\%$ (471 mg, $1.05 \mathrm{mmol}$ ). M.p. 109.4-111.2 ${ }^{\circ} \mathrm{C} ; \mathrm{R}_{f}=0.32$ (PE-EtOAc, 3:1). ${ }^{1} \mathrm{H} \mathrm{NMR}\left(400 \mathrm{MHz}, \mathrm{CDCl}_{3}\right) \delta 8.75(\mathrm{~s}, 1 \mathrm{H}$, Py-4'-H), 8.35 (t, J 1.6 Hz, 1H, Ph-H-2'), 8.25 (dd, J 8.0, 1.2 Hz, 1H, Ph-H-4'), 7.87 (d, J 8.0 Hz, 1H, Ph-H-6'), 7.63 (t, J $8.0 \mathrm{~Hz}, 1 \mathrm{H}, \mathrm{Ph}-\mathrm{H}-5^{\prime}$ ), 4.82 (d, J $\left.5.6 \mathrm{~Hz}, 1 \mathrm{H}, \mathrm{H}-2\right), 4.421$ (q, J $\left.7.2 \mathrm{~Hz}, 2 \mathrm{H}, \mathrm{OC}_{2} \mathrm{CH}_{3}\right), 4.415(\mathrm{q}, J 7.2 \mathrm{~Hz}, 2 \mathrm{H}$, $\left.\mathrm{OC}_{2} \mathrm{CH}_{3}\right), 4.27$ (dt, J 8.8, $\left.5.6 \mathrm{~Hz}, 1 \mathrm{H}, \mathrm{H}-3\right), 4.00$ (dd, J 16.0, $\left.5.6 \mathrm{~Hz}, 1 \mathrm{H}, \mathrm{H}-4\right), 3.92(\mathrm{dd}, J 16.0,7.2 \mathrm{~Hz}, 1 \mathrm{H}, \mathrm{H}-4)$, $2.88\left(\mathrm{~s}, 3 \mathrm{H}, \mathrm{Py}-\mathrm{CH}_{3}-6^{\prime}\right), 1.43\left(\mathrm{t}, J 7.2 \mathrm{~Hz}, 3 \mathrm{H}, \mathrm{OCH}_{2} \mathrm{C}_{3}\right), 1.42\left(\mathrm{t} J,=7.2 \mathrm{~Hz}, 3 \mathrm{H}, \mathrm{OCH}_{2} \mathrm{C}_{3}\right) .{ }^{13} \mathrm{C} \mathrm{NMR}(100 \mathrm{MHz}$, $\left.\mathrm{CDCl}_{3}\right) \delta 165.5,165.2,162.7,159.3,148.5,141.7,139.2,134.5,130.3,124.4,123.9,123.6,123.5,112.0,111.5$, 62.1, 61.9, 44.1, 37.6, 28.7, 25.1, 14.31, 14.29. IR (KBr) $v_{\max } 3089,2984,2907,2256,1716,1595,1533 \mathrm{~cm}^{-1}$. HRMS-TOF: calcd for $\mathrm{C}_{23} \mathrm{H}_{23} \mathrm{~N}_{4} \mathrm{O}_{6}[\mathrm{M}+\mathrm{H}]^{+} 451.1612$, found: 451.1611 .

2-Cyano-4-[2-(3,5-diethoxycarbonyl-6-methyl)pyridyl]-3-(2-nitrophenyl)butyronitrile (3f). Yellow oil, $47 \%$ (237 mg, $0.53 \mathrm{mmol}) . \mathrm{R}_{f}=0.31$ (PE-EtOAc, 4:1). ${ }^{1} \mathrm{H}$ NMR $\left(400 \mathrm{MHz}, \mathrm{CDCl}_{3}\right) \delta 8.67$ (s, $\left.1 \mathrm{H}, \mathrm{Py}-\mathrm{H}-4^{\prime}\right), 7.89$ (dd, J 8.0, 1.2 Hz, 1H, Ph-H-3'), 7.74 (d, J 7.2 Hz, 1H, Ph-H-6'), 7.60 (td, J 7.6, 1.2 Hz, 1H, Ph-H-4'), 7.48-7.44 (m, 1H, Ph-H-5'), 4.93 (dt, J 8.8, 5.6z, 1H, H-3), 4.73 (d, J $4.8 \mathrm{~Hz}, 1 \mathrm{H}, \mathrm{H}-2$ ), 4.43 (qd, J 7.2, 1.6 Hz, 2H, OC${ }_{2} \mathrm{CH}_{3}$ ), 4.37 (q, J $7.2 \mathrm{~Hz}, 2 \mathrm{H}, \mathrm{OCH}_{2} \mathrm{CH}_{3}$ ), 4.28 (dd, J 16.8, $\left.9.6 \mathrm{~Hz}, 1 \mathrm{H}, \mathrm{H}-4\right), 3.92(\mathrm{dd}, J 16.8,5.6 \mathrm{~Hz}, 1 \mathrm{H}, \mathrm{H}-4), 2.68\left(\mathrm{~s}, 3 \mathrm{H}, \mathrm{Py}-\mathrm{CH}_{3}-\right.$ $\left.6^{\prime}\right), 1.44\left(\mathrm{t}, J 7.2 \mathrm{~Hz}, 3 \mathrm{H}, \mathrm{OCH}_{2} \mathrm{C}_{3}\right), 1.39\left(\mathrm{t}, J 7.2 \mathrm{~Hz}, 3 \mathrm{H}, \mathrm{OCH}_{2} \underline{\mathrm{C}}_{3}\right) .{ }^{13} \mathrm{C} \mathrm{NMR}\left(100 \mathrm{MHz}, \mathrm{CDCl}_{3}\right) \delta 165.4,165.1$, $162.4,158.8,150.3,141.2,133.4,132.1,129.3,128.4,124.0,123.9,123.0,112.0,111.7,61.8,61.6,37.8,36.7$, 28.4, 24.4, 14.2, 14.1. IR (KBr) $v_{\max } 2984,2908,2257,1716,1596,1532 \mathrm{~cm}^{-1}$. HRMS-TOF: calcd for $\mathrm{C}_{23} \mathrm{H}_{23} \mathrm{~N}_{4} \mathrm{O}_{6}$ $[\mathrm{M}+\mathrm{H}]^{+} 451.1612$, found: 451.1607 .

2-Cyano-3-[2-(3,5-diethoxycarbonyl-6-methyl)pyridyl]methyl-5-phenyl-4-pentenonitrile (3g). Yellow oil, $19 \%$ (102 mg, $0.24 \mathrm{mmol}$ ); $21 \%$ (113 mg, $0.26 \mathrm{mmol}$ ) by Method B. R $=0.39$ (PE-EtOAc, 3:1). ${ }^{1} \mathrm{H} \mathrm{NMR}(400 \mathrm{MHz}$, $\left.\mathrm{CDCl}_{3}\right) \delta 8.74\left(\mathrm{~s}, 1 \mathrm{H}, \mathrm{Py}-\mathrm{H}-4^{\prime}\right), 7.40-7.27(\mathrm{~m}, 5 \mathrm{H}, \mathrm{Ph}-\mathrm{H}), 6.68(\mathrm{~d}, J 15.6 \mathrm{~Hz}, 1 \mathrm{H}, \mathrm{H}-5), 6.27(\mathrm{dd}, J 15.6,9.2 \mathrm{~Hz}, 1 \mathrm{H}$, $\mathrm{H}-4), 4.58(\mathrm{~d}, J 4.4 \mathrm{~Hz}, 1 \mathrm{H}, \mathrm{H}-2), 4.42$ (q, J $\left.7.2 \mathrm{~Hz}, 2 \mathrm{H}, \mathrm{OC}_{2} \mathrm{CH}_{3}\right), 4.40$ (qd, J 7.2, $0.4 \mathrm{~Hz}, 2 \mathrm{H}, \mathrm{OCH}_{2} \mathrm{CH}_{3}$ ), 3.77-3.74 (m, $1 \mathrm{H}, \mathrm{H}-3), 3.63-3.55$ (m, 2H, Py-H-2'), 2.90 (s, 3H, Py- $\left.\mathrm{CH}_{3}-6^{\prime}\right), 1.42\left(\mathrm{t}, J 7.2 \mathrm{~Hz}, 3 \mathrm{H}, \mathrm{OCH}_{2} \mathrm{C}_{3}\right.$ ), 1.41 (t, J 7.2 $\left.\mathrm{Hz}, 3 \mathrm{H}, \mathrm{OCH}_{2} \mathrm{CH}_{3}\right) .{ }^{13} \mathrm{C} N M R\left(100 \mathrm{MHz}, \mathrm{CDCl}_{3}\right) \delta 165.6,165.3,162.5,160.2,141.5,135.8,128.7,128.5,126.8$, $124.5,124.2,123.7,112.5,111.8,62.0,61.8,43.4,37.9,28.0,25.2,14.3$. IR (KBr) $v_{\max } 2983,2909,2254,1721$, 1595, $1552 \mathrm{~cm}^{-1}$. HRMS-TOF: calcd for $\mathrm{C}_{25} \mathrm{H}_{26} \mathrm{~N}_{3} \mathrm{O}_{4}[\mathrm{M}+\mathrm{H}]^{+} 432.1918$, found: 432.1917.

2-Cyano-4-[2-(3,5-diethoxycarbonyl-6-methyl)pyridyl]-3-(2-furanyl)butyronitrile (3h). Yellow oil, 10 \% (39 mg, $0.10 \mathrm{mmol}$ ); $87 \%$ (330 mg, $0.83 \mathrm{mmol}$ ) by Method B. $\mathrm{R}_{f}=0.43$ (PE-EtOAc, 3:1). ${ }^{1} \mathrm{H}$ NMR $\left(400 \mathrm{MHz}^{\mathrm{CDCl}}{ }_{3}\right)$ $\delta 8.74\left(\mathrm{~s}, 1 \mathrm{H}, \mathrm{Py}-\mathrm{H}-4^{\prime}\right), 7.43$ (dd, J 2.0, $0.8 \mathrm{~Hz}, 1 \mathrm{H}$, Furan-H-5'), 6.40 (d, J $3.2 \mathrm{~Hz}, 1 \mathrm{H}$, Furan-H-3'), 6.36 (dd, J 3.2 , $2.0 \mathrm{~Hz}, 1 \mathrm{H}$, Furan-H-4'), $4.70(\mathrm{~d}, J 5.2 \mathrm{~Hz}, 1 \mathrm{H}, \mathrm{H}-2), 4.42\left(\mathrm{q}, J 7.2 \mathrm{~Hz}, 2 \mathrm{H}, \mathrm{OC}_{2} \mathrm{CH}_{3}\right), 4.41(\mathrm{q}, J 7.2 \mathrm{~Hz}, 2 \mathrm{H}$, $\left.\mathrm{OCH}_{2} \mathrm{CH}_{3}\right), 4.30(\mathrm{dt}, J$ 8.4, $5.6 \mathrm{~Hz}, 1 \mathrm{H}, \mathrm{H}-3), 3.95$ (dd, J 16.0, $\left.6.0 \mathrm{~Hz}, 1 \mathrm{H}, \mathrm{H}-4\right), 3.80$ (dd, J 16.0, 8.8 Hz, 1H, H-4), 2.88 (s, 3H, Py-Me-6'), 1.43 (t, J $7.2 \mathrm{~Hz}, 3 \mathrm{H}, \mathrm{OCH}_{2} \mathrm{C}_{3}$ ), 1.42 (t, J $\left.7.2 \mathrm{~Hz}, 3 \mathrm{H}, \mathrm{OCH}_{2} \mathrm{CH}_{3}\right) .{ }^{13} \mathrm{C} \mathrm{NMR}(100 \mathrm{MHz}$, $\left.\mathrm{CDCl}_{3}\right) \delta 165.4,165.2,162.4,159.4,150.3,143.0,141.4,124.17,123.5,112.1,111.6,110.6,108.8,61.9,61.6$, 38.7, 36.4, 27.2, 25.0, 14.19, 14.15. IR (KBr) $v_{\max } 2984,2908,2256,1721,1595,1553 \mathrm{~cm}^{-1}$. HRMS-TOF: calcd for $\mathrm{C}_{21} \mathrm{H}_{22} \mathrm{~N}_{3} \mathrm{O}_{5}[\mathrm{M}+\mathrm{H}]^{+} 396.1554$, found: 396.1553 .

2-Cyano-4-[2-(3,5-diethoxycarbonyl-6-methyl)pyridyl]-3-(2-thienyl)butyronitrile (3i). Off-white oil, $23 \%$ (88 $\mathrm{mg}, 0.21 \mathrm{mmol}$ ); $39 \%\left(210 \mathrm{mg}, 0.51 \mathrm{mmol}\right.$ ) by Method B. $\mathrm{R}_{f}=0.37$ (petroleum ether-EtOAc, 4:1). ${ }^{1} \mathrm{H}$ NMR (400 $\left.\mathrm{MHz}, \mathrm{CDCl}_{3}\right) \delta 8.75\left(\mathrm{~s}, 1 \mathrm{H}, \mathrm{Py}-\mathrm{H}-4^{\prime}\right), 7.30(\mathrm{dd}, J 4.8,1.2 \mathrm{~Hz}, 1 \mathrm{H}$, Thiophene-H-5'), $7.22(\mathrm{~d}, J 3.2 \mathrm{~Hz}, 1 \mathrm{H}$, Thiophene-H-3'), 7.03 (dd, J 4.8, 3.6 Hz, 1H, Thiophene-H-4'), 4.80 (d, J 5.2 Hz, 1H, H-2), 4.47-4.38 (m, 1H, H-3), 
4.42 (q, J $7.2 \mathrm{~Hz}, 2 \mathrm{H}, \mathrm{OC}_{2} \mathrm{CH}_{3}$ ), 4.41 (q, J $7.2 \mathrm{~Hz}, 2 \mathrm{H}, \mathrm{OC}_{2} \mathrm{CH}_{3}$ ), 4.02 (dd, J 16.4, $5.2 \mathrm{~Hz}, 1 \mathrm{H}, \mathrm{H}-4$ ), 3.89 (dd, J 16.4, 9.2 Hz, 1H, H-4), 2.91 (s, 3H, Py-Me-6'), 1.43 (t, J 7.2 Hz, 3H, OCH $\mathrm{C}_{3}$ ), $1.42\left(\mathrm{t}, J 7.2 \mathrm{~Hz}, 3 \mathrm{H}, \mathrm{OCH}_{2} \mathrm{C}_{3}\right) .{ }^{13} \mathrm{C}$ NMR $\left(100 \mathrm{MHz}_{\mathrm{CDCl}}\right) \delta 165.5,165.2,162.5,159.4,141.5,139.4,127.2,126.8,125.7,124.1,123.6,112.3$, 111.7, 61.9, 61.7, 40.1, 39.0, 29.6, 25.1, 14.3, 14.2. IR (KBr) $v_{\max } 3110,2984,2906,2256,2227,1716,1595$, $1553 \mathrm{~cm}^{-1}$. HRMS-TOF: calcd for $\mathrm{C}_{21} \mathrm{H}_{22} \mathrm{~N}_{3} \mathrm{O}_{4} \mathrm{~S}[\mathrm{M}+\mathrm{H}]^{+} 412.1326$, found: 412.1322 .

2-Cyano-3-[2-(3,5-diethoxycarbonyl-6-methyl)pyridyl]methyl-4,4-dimethylvaleronitrile (3j). Colorless liquid, $26 \%$ (107 mg, $0.28 \mathrm{mmol}$ ). $\mathrm{R}_{f}=0.49$ (petroleum ether-EtOAc, 4:1). ${ }^{1} \mathrm{H} \mathrm{NMR}\left(400 \mathrm{MHz}, \mathrm{CDCl}_{3}\right) \delta 8.75(\mathrm{~s}, 1 \mathrm{H}, \mathrm{Py}$ $\mathrm{H}-4^{\prime}$ ), 4.44-4.39 (m, 4H, OC$\left.{ }_{2} \mathrm{CH}_{3}\right), 4.32$ (d, J $\left.2.4 \mathrm{~Hz}, 1 \mathrm{H}, \mathrm{H}-2\right), 3.78$ (dd, J 16.0, $\left.2.8 \mathrm{~Hz}, 1 \mathrm{H}, \mathrm{H}-4\right), 3.38$ (dd, J 16.0, $11.2 \mathrm{~Hz}, 1 \mathrm{H}, \mathrm{H}-4), 2.92$ (s, 3H, Py-Me-6'), 2.84 (dt, J 10.8, $2.8 \mathrm{~Hz}, 1 \mathrm{H}, \mathrm{H}-3$ ), 1.429 (t, J 7.2 Hz, 3H, OCH $\mathrm{CH}_{3}$ ), $1.425\left(\mathrm{t}, J 7.2 \mathrm{~Hz}, 3 \mathrm{H}, \mathrm{OCH}_{2} \mathrm{C}_{3}\right), 1.18(\mathrm{~s}, 9 \mathrm{H}, t-\mathrm{Bu}) .{ }^{13} \mathrm{C} \mathrm{NMR}\left(100 \mathrm{MHz}, \mathrm{CDCl}_{3}\right) \delta 165.7,165.4,162.6,161.5$, 141.6, 123.9, 123.4, 113.5, 61.7, 61.6, 48.5, 34.7, 34.5, 27.9, 25.0, 22.9, 14.3. IR (KBr) $v_{\max } 2971,2252,1723$, 1596, $1552 \mathrm{~cm}^{-1}$. HRMS-TOF: calcd for $\mathrm{C}_{21} \mathrm{H}_{28} \mathrm{~N}_{3} \mathrm{O}_{4}[\mathrm{M}+\mathrm{H}]^{+} 386.2074$, found: 386.2070 .

2-Cyano-4-[2-(3,5-diethoxycarbonyl-4,6-dimethyl)pyridyl]-3-phenylbutyronitrile (3k). Brown oil, 52 \% (142 $\mathrm{mg}, 0.34 \mathrm{mmol}$ ). $\mathrm{R}_{f}=0.30$ (petroleum ether-EtOAc, 4:1). ${ }^{1} \mathrm{H} \mathrm{NMR}\left(400 \mathrm{MHz}, \mathrm{CDCl}_{3}\right) \delta 7.43-7.38(\mathrm{~m}, 5 \mathrm{H}, \mathrm{Ph}-\mathrm{H})$, 4.74 (d, J $4.4 \mathrm{~Hz}, 1 \mathrm{H}, \mathrm{H}-2$ ), 4.44 (q, J $7.2 \mathrm{~Hz}, 2 \mathrm{H}, \mathrm{Py}-\mathrm{CO}_{2} \mathrm{C}_{2} \mathrm{CH}_{3}-5^{\prime}$ ), 4.39 (q, J 7.2 Hz, 2H, Py-CO $\left.\mathrm{C}_{2} \mathrm{CH}_{3}-3^{\prime}\right), 3.96$ (dt, J 10.0, 4.4 Hz, 1H, H-3), 3.45 (dd, J 15.6, 10.0 Hz, 1H, H-4), 3.27 (dd, J 15.6, $4.4 \mathrm{~Hz}, 1 \mathrm{H}, \mathrm{H}-4), 2.55$ (s, 3H, Py$\mathrm{CH}_{3}-6^{\prime}$ ), 2.28 (s, 3H, Py-CH$-4^{\prime}$ ), 1.41 (t, J 7.2 Hz, 3H, Py- $\left.\mathrm{CO}_{2} \mathrm{CH}_{2} \mathrm{C}_{3}-5^{\prime}\right), 1.34$ (t, J 7.2 Hz, 3H, Py- $\left.\mathrm{CO}_{2} \mathrm{CH}_{2} \mathrm{C}_{3}-3^{\prime}\right)$. ${ }^{13} \mathrm{C} \mathrm{NMR}\left(100 \mathrm{MHz}_{1} \mathrm{CDCl}_{3}\right) \delta 167.9,167.6,155.5,153.5,143.0,137.0,129.1,128.9,128.8,128.2,128.1,112.5$, $111.8,62.1,61.9,44.6,36.9,28.7,23.0,17.2,14.2,14.1 . \mathrm{IR}(\mathrm{KBr}) v_{\max } 2983,2255,1735,1561 \mathrm{~cm}^{-1}$. HRMS-TOF calcd for $\mathrm{C}_{24} \mathrm{H}_{26} \mathrm{~N}_{3} \mathrm{O}_{4}[\mathrm{M}+\mathrm{H}]^{+} 420.1918$, found: 420.1914 .

2-Cyano-4-[2-(3,5-diethoxycarbonyl-6-methyl-4-phenyl)pyridyl]-3-phenylbutyronitrile (3I). Off-white oil, 77 $\%(223 \mathrm{mg}, 0.46 \mathrm{mmol}) . \mathrm{R}_{f}=0.44$ (petroleum ether-EtOAc, 3:1). ${ }^{1} \mathrm{H} \mathrm{NMR}\left(400 \mathrm{MHz}, \mathrm{CDCl}_{3}\right) \delta 7.46-7.36(\mathrm{~m}, 8 \mathrm{H}$, Ph-H), 7.24-7.22 (m, 2H, Ph-H), 4.77 (d, J $4.8 \mathrm{~Hz}, 1 \mathrm{H}, \mathrm{H}-2), 4.06-4.00(\mathrm{~m}, 1 \mathrm{H}, \mathrm{H}-3), 4.02$ (q, J 7.2 Hz, 2H, Py$\mathrm{CO}_{2} \underline{\mathrm{C}}_{2} \mathrm{CH}_{3}-5^{\prime}$ ), 3.96 (q, J 7.2 Hz, 2H, Py- $\mathrm{CO}_{2} \mathrm{C}_{2} \mathrm{CH}_{3}-3^{\prime}$ ), 3.54 (dd, J 16.0, $10.0 \mathrm{~Hz}, 1 \mathrm{H}, \mathrm{H}-4$ ), 3.38 (dd, J 16.0, 4.4 $\mathrm{Hz}, 1 \mathrm{H}, \mathrm{H}-4), 2.64$ (s, 3H, Py- $\mathrm{CH}_{3}-6^{\prime}$ ), 0.91 (t, J 7.2 Hz, 3H, Py- $\left.\mathrm{CO}_{2} \mathrm{CH}_{2} \mathrm{CH}_{3}-5^{\prime}\right), 0.81$ (t, J 7.2 Hz, 3H, $\mathrm{Py}_{-} \mathrm{CO}_{2} \mathrm{CH}_{2} \mathrm{CH}_{3}-$ $\left.3^{\prime}\right) .{ }^{13} \mathrm{C}$ NMR $\left(100 \mathrm{MHz} \mathrm{CDCl}_{3}\right) \delta 167.5,167.3,156.1,154.1,147.1,137.1,136.2,129.2,129.0,128.8,128.3$, $128.2,128.1,127.7,112.5,111.9,61.9,61.7,44.8,37.1,28.8,23.2,13.6,13.5 . \mathrm{IR}(\mathrm{KBr}) v_{\max } 2982,2254,1724$, $1557 \mathrm{~cm}^{-1}$. ESI-MS m/z 504.6 [M + Na] $]^{+}$. HRMS-ESI calcd for $\mathrm{C}_{29} \mathrm{H}_{28} \mathrm{~N}_{3} \mathrm{O}_{4}[\mathrm{M}+\mathrm{H}]^{+}$482.2074, found: 482.2076 .

2-Cyano-4-[2-(6-methyl)pyridyl]-3-phenylbutyronitrile (5a). ${ }^{14}$ Green oil, $62 \%(229 \mathrm{mg}, 0.88 \mathrm{mmol}) . \mathrm{R}_{f}=0.36$ (PE-EtOAc, 3:1). ${ }^{1} \mathrm{H}$ NMR (400 MHz, CDCl $) \delta 7.51\left(\mathrm{t}, J 7.6 \mathrm{~Hz}, 1 \mathrm{H}, \mathrm{Py}-\mathrm{H}-4^{\prime}\right), 7.44-7.36(\mathrm{~m}, 5 \mathrm{H}, \mathrm{Ph}-\mathrm{H}), 7.05(\mathrm{~d}, J$ $\left.7.6 \mathrm{~Hz}, 1 \mathrm{H}, \mathrm{Py}-\mathrm{H}-5^{\prime}\right), 6.95$ (d, J $\left.7.6 \mathrm{~Hz}, 1 \mathrm{H}, \mathrm{Py}-\mathrm{H}-3^{\prime}\right), 4.83$ (d, J $5.2 \mathrm{~Hz}, 1 \mathrm{H}, \mathrm{H}-2$ ), ${ }^{11} 3.91$ (dt, J 9.6, 5.2 Hz, 1H, H-3), 3.45 (dd, J 15.2, $10.0 \mathrm{~Hz}, 1 \mathrm{H}, \mathrm{H}-4),{ }^{11} 3.29$ (dd, J 15.2, $\left.4.8 \mathrm{~Hz}, 1 \mathrm{H}, \mathrm{H}-4\right),{ }^{11} 2.56\left(\mathrm{~s}, 3 \mathrm{H}, \mathrm{Py}-\mathrm{CH}_{3}-6^{\prime}\right) .{ }^{13} \mathrm{C} \mathrm{NMR}(100$ $\left.\mathrm{MHz}_{2} \mathrm{CDCl}_{3}\right) \delta 158.4,156.1,137.1,137.1,129.0,128.7,128.0,121.8,120.9,112.5,112.0,45.1,39.3,28.6,24.4$. IR $(\mathrm{KBr}) v_{\max } 2903,2255,2205,1967,1883,1652,1594,1576 \mathrm{~cm}^{-1} . \mathrm{ESI}-\mathrm{MS} \mathrm{m} / \mathrm{z} 284.2\left([\mathrm{M}+\mathrm{Na}]^{+}\right), 196.1([\mathrm{M}-$ $\left.\left.\mathrm{CH}_{2}(\mathrm{CN})_{2}+\mathrm{H}\right]^{+}\right)$. HRMS-TOF calcd for $\mathrm{C}_{17} \mathrm{H}_{16} \mathrm{~N}_{3}[\mathrm{M}+\mathrm{H}]^{+}$262.1339, found: 262.1336.

2-Cyano-3-phenyl-4-(4-pyridyl)butyronitrile (5b). Pale yellow oil, $12 \%$ (33 mg, $0.13 \mathrm{mmol}$ ). $\mathrm{R}_{f}=0.59$ (EtOAc). ${ }^{1} \mathrm{H}$ NMR $\left(400 \mathrm{MHz} \mathrm{CDCl}_{3}\right.$ ) $\delta 8.48$ (d, J $\left.6.0 \mathrm{~Hz}, 2 \mathrm{H}, \mathrm{Py}-\mathrm{H}-2^{\prime} \& \mathrm{Py}-\mathrm{H}-6{ }^{\prime}\right), 7.42-7.37$ (m, 3H, Ph-H-3', Ph-H-4'\&Ph-H5'), 7.32-7.30 (m, 2H, Py-H-2'\&Py-H-6'), 7.04 (d, J 6.0 Hz, 2H, Py-H-3'\&Py-H-5'), 3.98 (d, J 5.6 Hz, 1H, H-2), 3.52 (dt, J 8.8, 6.4 Hz, 1H, H-3), 3.33 (dd, J 14.0, 6.8 Hz, 1H, H-4), 3.25 (dd, J 14.0, 8.8 Hz, 1H, H-4). ${ }^{13} \mathrm{C} \mathrm{NMR} \mathrm{(100}$ $\left.\mathrm{MHz}_{2} \mathrm{CDCl}_{3}\right) \delta 150.2,145.7,135.4,129.5,129.5,127.9,124.3,111.7,111.5,47.3,37.8,29.4 . \mathrm{IR}(\mathrm{KBr}) v_{\max } 3070$, 3034, 2929, 2803, 2251, 2216, 1603, $1559 \mathrm{~cm}^{-1}$. HRMS-TOF calcd for $\mathrm{C}_{16} \mathrm{H}_{14} \mathrm{~N}_{3}\left([\mathrm{M}+\mathrm{H}]^{+}\right)$248.1182, found: 248.1181. 


\section{Acknowledgements}

This project is supported by the National Natural Science Foundation of China (No's 21572118 and 21572080) and Jiangsu University (No's 10JDG042 and 14JDG018).

\section{Supplementary Material}

${ }^{1}$ H NMR and ${ }^{13}$ C NMR data.

\section{References}

1. Drapak, I.; Perekhoda, L.; Tsapko, T.; Berezniakova, N.; Tsapko, Y. J. Heterocyclic Chem. 2017, 54, 21172128.

https://doi.org/10.1002/jhet.2837

2. Triggle, D. J. In Comprehensive Medicinal Chemistry; Hansch, C.; Sammes, P. G.; Taylor, J. B. Eds.; Pergamon: Oxford, 1990; Vol. 3, pp 1047-1099.

3. Lemke, T. L.; Williams, D. A.; Roche, V. F.; Zito, S. W. In Foye's Principles of Medicinal Chemistry, 6th Edn.; Lippincott Williams \& Wilkins: USA, 2008; pp 710-711.

4. Hantzsch, A. Chem. Ber. 1881, 14, 1637-1638.

https://doi.org/10.1002/cber.18810140214

5. Saini, A.; Kumar, S.; Sandhu, J. S. J. Sci. Ind. Res. 2008, 67, 95-111.

https://doi.org/10.1016/B978-0-12-408100-0.00006-9

6. Comins, D. L.; Higuchi, K.; Young, D. W. In Advances in Heterocyclic Chemistry; Katritzky, A. R. Ed.; Elsevier, 2013; Vol. 110, pp 175-235.

https://doi.org/10.1016/B978-0-12-408100-0.00006-9

7. Chang, L.; Lai, J.; Yuan, G. Chin. J. Chem. 2016, 34, 887-894.

https://doi.org/10.1002/cjoc.201600255

8. Rao, H. S. P.; Parthiban, A. Org. Biomol. Chem. 2014, 12, 6223-6238. https://doi.org/10.1039/C4OB00628C

9. Sueki, S.; Takei, R.; Zaitsu, Y.; Abe, J.; Fukuda, A.; Seto, K.; Furukawa, Y.; Shimizu, I. Eur. J. Org. Chem. 2014, 5281-5301.

https://doi.org/10.1002/ejoc.201402426

10. Booker, E.; Eisner, U. J. Chem. Soc., Perkin Trans. 1 1975, 929-931.

https://doi.org/10.1039/p19750000929

11. Singh, S.; Sharma, V. K.; Gill, S.; Sahota, R. I. K. J. Chem. Soc., Perkin Trans. 1 1985, 437-440.

https://doi.org/10.1039/p19850000437

12. Chen, Q.-A.; Chen, M.-W.; Yu, C.-B.; Shi, L.; Wang, D.-S.; Yang, Y.; Zhou, Y.-G. J. Am. Chem. Soc. 2011, 133, 16432-16435.

https://doi.org/10.1021/ja208073w

13. Kumar, N. S.; Rao, L. C.; Jagadeesh Babu, N.; Kumar, V. D.; Murthy, U. S. N.; Meshram, H. M. Synlett 2015, 26, 1808-1814. 
https://doi.org/10.1055/s-0034-1380212

14. Mallepalli, R.; Vennam, D. K. R.; Perali, R. S. Tetrahedron Lett. 2016, 57, 4541-4543.

https://doi.org/10.1016/j.tetlet.2016.08.083

15. Campaigne, E.; Bulbenko, G. F.; Kreighbsum, W. E.; Maulding, D. R. J. Org. Chem. 1962, 27, 4428-4432. https://doi.org/10.1021/j001059a074

16. Campaigne, E.; Maulding, D. R.; Roelofs, W. R. J. Org. Chem. 1964, 29, 1543-1549. https://doi.org/10.1021/jo01029a066

17. Campaigne, E.; Roelofs, W. R. J. Org. Chem. 1965, 30, 396-400. https://doi.org/10.1021/jo01013a021

18. Campaigne, E.; Ellis, R. L. Chem. Commun. (London) 1966, 140-141. https://doi.org/10.1039/c1966000140b

This paper is an open access article distributed under the terms of the Creative Commons Attribution (CC BY) license (http://creativecommons.org/licenses/by/4.0/ 